\title{
Tartary Buckwheat R2R3-MYB Gene FtMYB3 Negatively Regulates Anthocyanin and Proanthocyanin Biosynthesis
}

\author{
Lei Wang ${ }^{\dagger}$, Renyu Deng ${ }^{+}{ }^{-}$, Yuechen Bai, Huala Wu, Chenglei Li, Qi Wu and Haixia Zhao *
}

check for updates

Citation: Wang, L.; Deng, R.; Bai, Y.; Wu, H.; Li, C.; Wu, Q.; Zhao, H. Tartary Buckwheat R2R3-MYB Gene FtMYB3 Negatively Regulates Anthocyanin and Proanthocyanin Biosynthesis. Int. J. Mol. Sci. 2022, 23, 2775. https://doi.org/10.3390/ ijms23052775

Academic Editor:

Endang Septiningsih

Received: 1 November 2021

Accepted: 6 January 2022

Published: 3 March 2022

Publisher's Note: MDPI stays neutral with regard to jurisdictional claims in published maps and institutional affiliations.

Copyright: (C) 2022 by the authors. Licensee MDPI, Basel, Switzerland. This article is an open access article distributed under the terms and conditions of the Creative Commons Attribution (CC BY) license (https:// creativecommons.org/licenses/by/ $4.0 /)$.
College of Life Science, Sichuan Agricultural University, No. 46, Xinkang Road, Ya'an 625014, China; leiwang@sicau.edu.cn (L.W.); dengrenyu@caas.cn (R.D.); ybai@ice.mpg.de (Y.B.); hualawu@sicau.edu.cn (H.W.); 13981@sicau.edu.cn (C.L.); wuqi@sicau.edu.cn (Q.W.)

* Correspondence: zhaohaixia@sicau.edu.cn; Tel.: +86-13458850051

+ These authors contributed equally to this work.

\begin{abstract}
Anthocyanins and proanthocyanidins (PAs) are vital secondary metabolites in Tartary buckwheat because of their antioxidant capacities and radical scavenging functions. It has been demonstrated that R2R3-MYB transcription factors (TFs) are essential regulators of anthocyanin and PA biosynthesis in many plants. However, their regulatory mechanisms in Tartary buckwheat remain to be clarified. Here, we confirmed the role of FtMYB3 in anthocyanin and PA biosynthesis. FtMYB3, which belongs to the subgroup 4 R2R3 family was predominantly expressed in roots. The transcriptional expression of FtMYB3 increased significantly under hormone treatment with SA and MeJA and abiotic stresses including drought, salt, and cold at the seedling stage. Functional analyses showed that FtMYB3 negatively regulated anthocyanin and PA biosynthesis, primarily via downregulating the expression of the DFR, ANS, BAN, and TT13 in transgenic Arabidopsis thaliana, which may depend on the interaction between FtMYB3 and FtbHLH/FtWD40. Altogether, this study reveals that FtMYB3 is a negative regulatory transcription factor for anthocyanin and PA biosynthesis in Tartary buckwheat.
\end{abstract}

Keywords: anthocyanin; FtMYB3; proanthocyanidin; Tartary buckwheat; transcription factor

\section{Introduction}

Anthocyanins and proanthocyanidins (PAs) are derived from phenylalanine and mainly distributed in the fruit, bark, leaves and seeds of plants [1]. They have multiple functions in plants, including attraction of pollinators, protection against UV light damage and oxidative stress, regulation of auxin transport and allelopathy [2,3]. Moreover, PAs and anthocyanins show different health effects in vitro, such as anticancer and antiinflammatory activities, as well as antioxidation $[4,5]$. Therefore, further exploring their synthesis and regulatory mechanisms is essential.

To date, increasing evidence suggests that the anthocyanin and PA biosynthetic pathway is well-conserved in monocots and dicots, and most steps are the same [6,7]. Firstly, leucoanthocyanidins are converted into unstable anthocyanidins by anthocyanidin synthase (ANS). Then, anthocyanidins are either immediately glycosylated to anthocyanins by anthocyanidin 3-O-glycosyltransferases (UGTs) or reduced to flavan-3-ols by anthocyanidin reductase (ANR) for PA biosynthesis [8]. Previous studies have elucidated that the above process is mainly regulated by TFs such as MYB, bHLH, WD40, and WRKY families [9-11]. Among these TFs, R2R3 MYB plays an indispensable role in regulating anthocyanin and PA biosynthesis, which have been studied extensively in different species. For example, several activators, including PcMYB114, McMYB12, MdMYB9/11, co-regulate PA and anthocyanin biosynthesis [12-14]. In contrast, NtMYB3 and MdMYB16 inhibit anthocyanin biosynthesis via the typical conserved motifs $C 2$ at their C-terminus [15,16]. However, an R2R3-MYB TF, VviMYB86 positively regulated PA biosynthesis by upregulating the $L A R$ expression but repressed the anthocyanin biosynthesis with downregulation of the 
transcript levels of VviANS and VviUFGT [17]. These studies indicate that the molecular mechanism underlying anthocyanin and PA accumulation is complicated. Notably, the ternary complex of MYB proteins is also involved in the synthesis of anthocyanins and PAs in different plants $[18,19]$. Recently, two novel ternary complexes, the JAZ1-TRB1-MYB9 and MdHY5-MdWRKY41-MdMYB were found to dynamically modulate anthocyanin and PA accumulation in apple [20,21]. In addition, anthocyanin and PA biosynthesis are also influenced by environmental signals such as phytohormone signals, UV radiation, cold, drought and light [22,23]. Collectively, this strongly suggests that MYB TFs play a central role in anthocyanin and PA synthesis.

Tartary buckwheat (Fagopyrum tataricum), which belongs to the genus Fagopyrum in the Polygonaceae family, is widely recognized as a pseudo-cereal that possesses unparalleled nutritive value $[24,25]$. As a plant originating in high-altitude areas, abiotic stresses such as ultraviolet and drought have a significant effect on the growth of Tartary buckwheat. Studies have reported that the accumulation of anthocyanins and PAs has benefits for plant survival under these stresses [26-28]. Therefore, understanding the corresponding mechanisms of their biosynthesis and regulation in Tartary buckwheat has gained considerable attention. Recently, several positive and negative regulators were identified in Tartary buckwheat. The first functional characterization of R2R3-type MYB TFs from Tartary buckwheat, found that FtMYB1 and FtMYB2 control the PA accumulation [29]. Ectopic expression of FtMYB15 in Arabidopsis promotes the accumulation of anthocyanins and PA in leaves and seed coats [30]. The latest studies showed that FtMYB8 and FtMYB18 from the SG4-like MYB subfamily act as a negative regulator of anthocyanin/PA biosynthesis [31,32]. Additionally, our previous study showed that FtMYB3 interacted with the jasmonate-ZIM domain protein FtJAZ2 to influence anthocyanin biosynthesis in Tartary buckwheat [33]. Furthermore, FtMYB3 is likely to be involved in anthocyanin biosynthesis in the hairy roots of Tartary buckwheat [34]. However, the molecular mechanism of FtMYB3 mediating in which signaling pathways of the anthocyanin biosynthesis remains unclear. In this study, we validated that FtMYB3 acts as a negative regulator of anthocyanin and PA accumulation. This study sheds light on the regulatory mechanism of R2R3-MYB repressors on anthocyanin and PA synthesis.

\section{Results}

\subsection{Tartary Buckwheat FtMYB3 Is an SG4 R2R3 MYB Protein}

Based on the genomic database and our transcriptomic database (data not shown), FtMYB3 (GenBank ID: JF313349) was identified in Tartary buckwheat. The full-length cDNA of FtMYB3 is $624 \mathrm{bp}$ in size and encodes a putative protein of 207 amino acids with a predicted molecular weight of about $23.4 \mathrm{kDa}$ and a calculated isoelectric point (PI) of 8.19. Phylogenetic analysis indicated that FtMYB3 was phylogenetically related to AtMYB3 and MdMYB3 (Figure 1A), belonging to the subgroup 4 (SG4) R2R3 family of plant MYB TFs. These factors negatively regulate the $A$. thaliana phenylpropanoid biosynthesis and activate the apple anthocyanin accumulation, respectively $[35,36]$. Amino acid sequence alignments between FtMYB3 and other MYBs indicated that FtMYB3 consists of R2 and R3 DNAbinding domains at the $\mathrm{N}$ terminus and a bHLH motif $([\mathrm{D} / \mathrm{E}] \mathrm{L} \times 2[\mathrm{R} / \mathrm{K}] \times 3 \mathrm{~L} \times 6 \mathrm{~L} \times 3 \mathrm{R})$ within R3 [37] (Figure 1B). Moreover, FtMYB3 had a relatively short C-terminal region with two conserved motifs $\mathrm{C} 1$ and $\mathrm{C} 2$. These results suggest FtMYB3 is an SG4 R2R3 MYB protein that may function as a transcriptional regulator in the flavonoid pathway.

\subsection{Expression Pattern of FtMYB3 and Anthocyanin Content in Tartary Buckwheat}

To preliminarily understand the role of FtMYB3 in Tartary buckwheat, the expression pattern of FtMYB3 was examined by qRT-PCR in the roots, stems, leaves, flowers, and seeds of Xiqiao No. 2 at the flowering stage. As shown in Figure 2A, the expression of FtMYB3 was most highly expressed in roots, followed by stems and leaves. Differently, the lowest expression was exhibited in flowers and seeds, almost tending to 0 . Additionally, the total anthocyanin content of these tissues was tested. The anthocyanin content was 
determined in all tested tissues with a relatively high accumulation in flowers and stems (Figure 2B). Given that the tissue expression pattern of FtMYB3 is negatively correlated with anthocyanin accumulation $(\mathrm{R} 2=-0.3751)$, we speculated that FtMYB3 possibly inhibits anthocyanin synthesis in Tartary buckwheat.

A
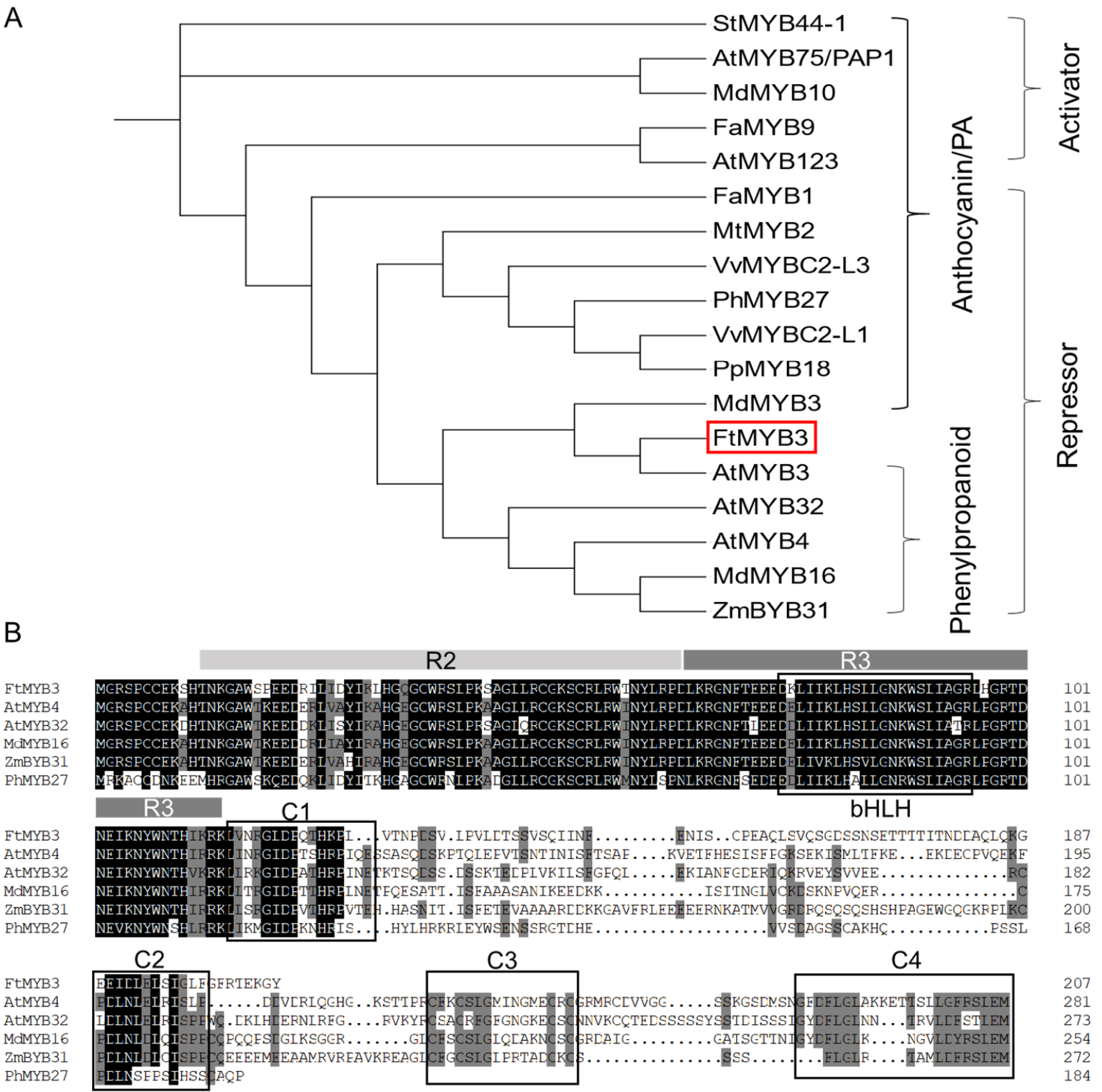

B

Figure 1. Molecular identification of FtMYB3. (A) Phylogenetic tree derived from amino acid sequences of R2R3-MYB TFs in Tartary buckwheat and other species. GenBank accession numbers are as follows: StMYB44-1 (QCH00894.1), AtMYB75 (Q9FE25.1), MdMYB10 (ACQ45201.1), FaMYB9 (JQ989281.1), AtMYB123 (Q9FJA2.1), FaMYB1 (AF401220), MtMYB2 (XP_003616388.1), VvMYBC2-L3 (KM046932), PhMYB27 (AHX24372.1), VvMYBC2-L1 (ABW34393), PpMYB8 (ACA33844.1), MdMYB3 (AEX08668.1), FtMYB3 (AEC32978.1), AtMYB3 (BAA21618.1), AtMYB32 (EFH43356.1), AtMYB4 (BAA21619), MdMYB16 (ADL36756.1), ZmMYB31 (NP_001105949). FtMYB3 is highlighted with a red box. (B) Multiple sequence alignments of MYB3. Conserved residues are highlighted in black and partial conservation is shown in grey. The R2 and R3 domains are indicated in greyish white and grey boxes, respectively. Conserved motifs in the $\mathrm{C}$ terminus are boxed and numbered as $\mathrm{C} 1 \sim \mathrm{C} 4$. Identical and similar amino acid residues are marked in black and grey, respectively. 
A

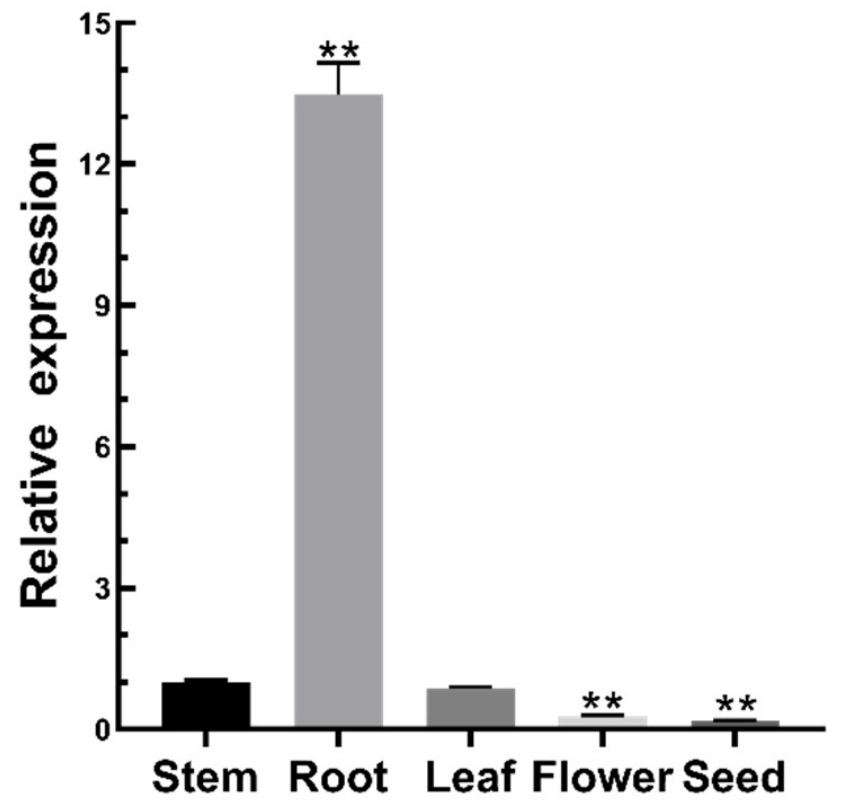

B

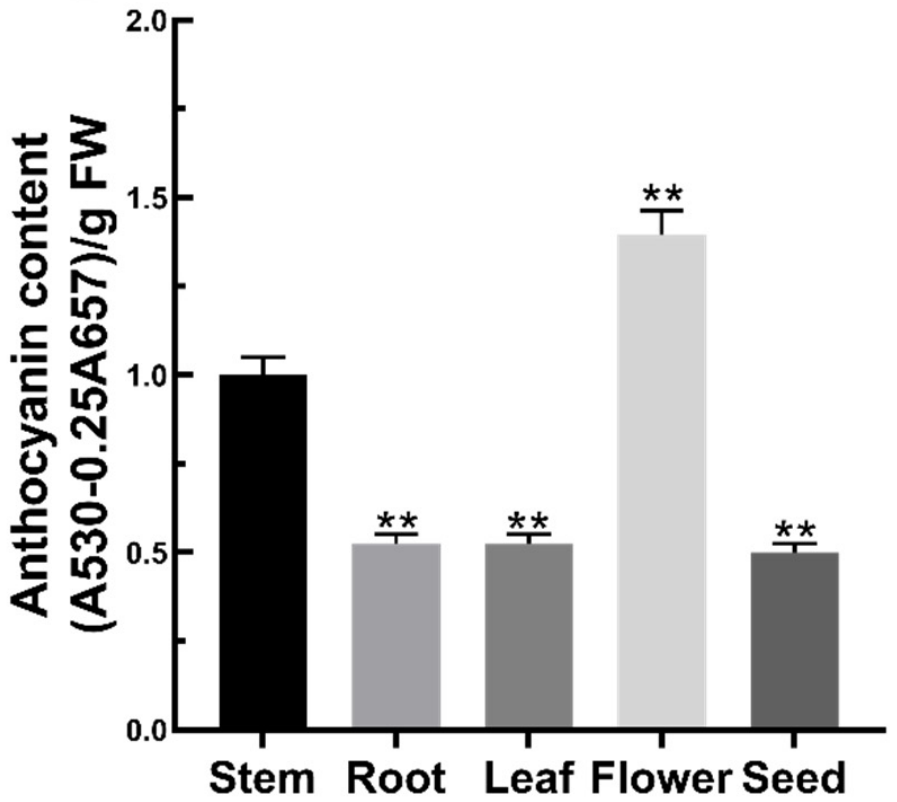

Figure 2. The transcription level of $F t M Y B 3$ and anthocyanin content in different organs. Tartary buckwheat roots, stems, leaves, flowers, and seeds were collected at 40 days after sowing for the analysis of gene expression pattern and the determination of anthocyanin content. (A) FtMYB3 expression level in different organs; (B) Anthocyanin content in various organs. The accumulation of anthocyanin content and gene expression level in stems are defined as " 1 ". ${ }^{* *}$ means extremely significant difference at $p<0.01$ level; Error bars reflect \pm SDs.

\subsection{Induction of FtMYB3 by Multiple Environmental Factors and Plant Hormones}

The transcription level of MYBs was affected by multiple factors, including environmental factors such as temperature, drought and salinity, as well as plant hormones, MeJA and SA $[9,38]$. To further examine the response of FtMYB3 to the external environment signals, we cloned its promoter starting from the site of $1986 \mathrm{bp}$ at the upstream of ATG start codon (Supplementary Materials, Text S1). Analysis of the cis-regulatory elements within this promoter showed that these elements were classified into two groups based on their responsive functions: environmental responsive elements including light (e.g., G-box, CACGAC), low temperature (e.g., LTR, CCGAAA), drought (e.g., MBS, CAACTG) and hormone-responsive elements including GA (GARE, TCTGTTG), an auxin (e.g., TGAelement, AACGAC), an SA (e.g., TCA-element, CCATCTTTTT) and MeJA (e.g., CGTCAmotif, CGTCA) (Table S1). Furthermore, several other types of cis-acting elements also exhibited many TATA box, CAAT box, and MYB binding sites (Table S1). These results suggest that distinct environmental factors and plant hormones can regulate the expression of FtMYB3.

qRT-PCR was used to analyze the gene expression pattern of FtMYB3 in Tartary buckwheat seedlings under different treatments. Under PEG6000 and NaCl treatments, FtMYB3 expression exhibited a gradually increasing pattern and reached the highest values of 6.82 and 7.19 at $15 \mathrm{~h}$ (Figure 3). FtMYB3 was also strongly induced by SA and MeJA $(p<0.01)$ (Figure 3). Furthermore, low temperature can significantly up-regulate FtMYB3 at $3 \mathrm{~h}$ and $6 \mathrm{~h}$. These results show that FtMYB3 may be involved in the stress responses to SA, MeJA, salt, cold and drought. 


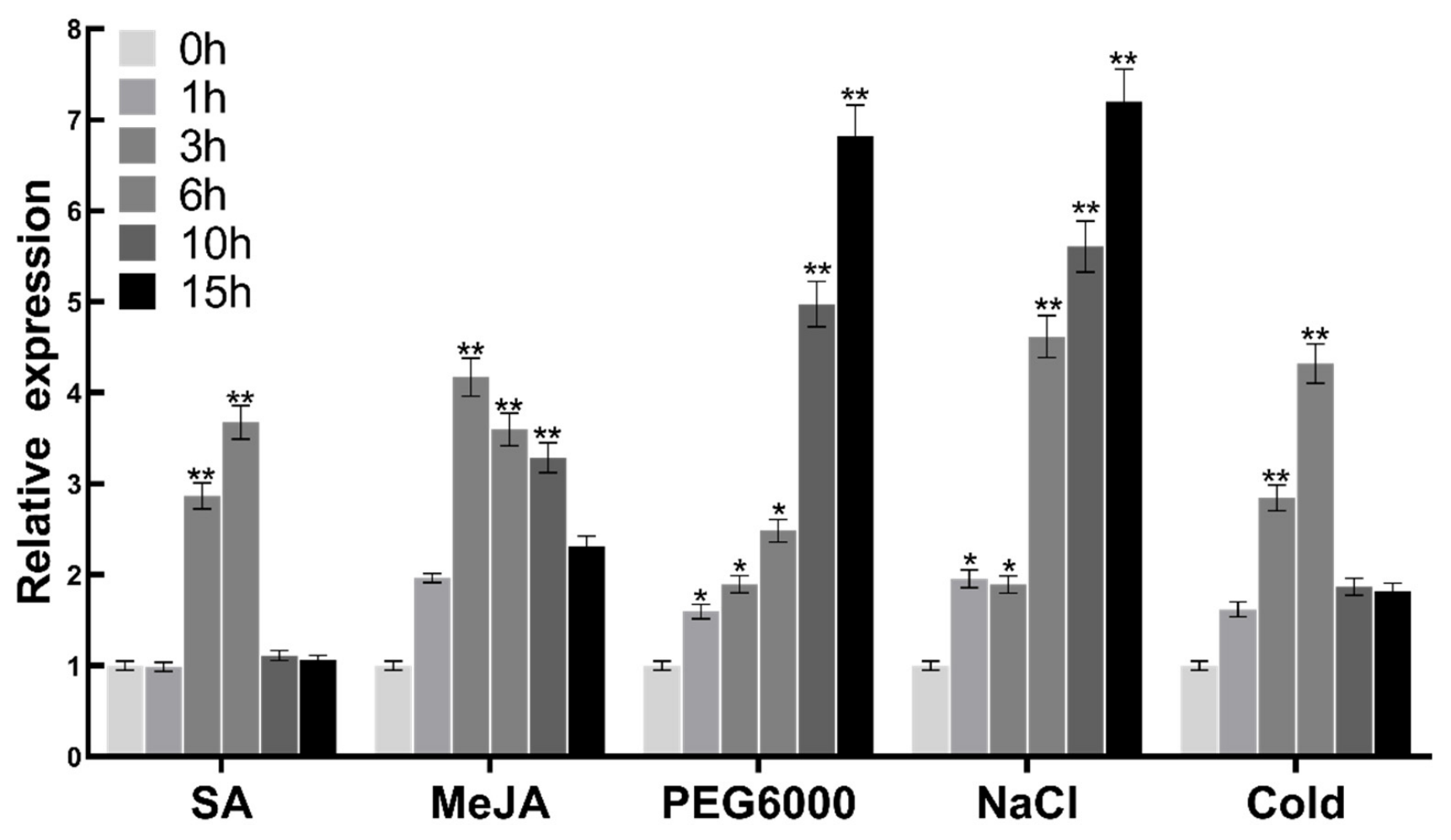

Figure 3. Expression of FtMYB3 in Tartary buckwheat under different treatments (1 mmol/L SA,

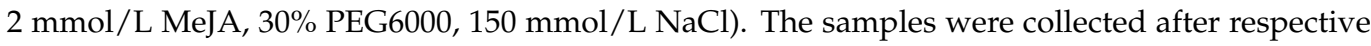
treatments for $0,1,3,6,10$, and $15 \mathrm{~h}$. The relative expression of FtMYB3 was analyzed by qPCR, and the expression level of FtMYB3 at $0 \mathrm{~h}$ was defined as 1 . The expression level was calculated from three replicates. Error bars denote \pm SDs. ${ }^{* *}$ means extremely significant difference at $p<0.01$ level; * means significant difference at $p<0.05$ level.

\subsection{FtMYB3 Inhibits Anthocyanin/PA Accumulation}

To explore whether FtMYB3 regulates anthocyanin synthesis, transgenic tobaccos (termed $N t \# 1, N t \# 2$ ) and A. arabidopsis (named At \#1, At \#2) overexpressing FtMYB3 were obtained. Results showed that the petals of lines $N t \# 1$ and $N t \# 2$ exhibited lighter pigmentation compared with that in wild-type (WT), indicating that the anthocyanin synthesis was inhibited (Figure 4A). Furthermore, the anthocyanin content of tobacco flowers overexpressing FtMYB3 was significantly decreased by $38.75 \%$ and $21.25 \%$ compared to that in WT (Figure 4B).

Similarly, the pigment deposition in seedlings of At\#1 and At \#2 was less than that in Arabidopsis WT (named Col-0) (Figure 4C). Moreover, the color of the seed coats was obviously lighter in At \#1 and At \#2 than in Col-0, indicating weaker pigmentation in plants overexpressing FtMYB3 (Figure 4D). The above results suggest that FtMYB3 may also affect the synthesis of other secondary metabolites. Therefore, the contents of anthocyanin, PA and rutin was determined in transgenic Arabidopsis. Figure 4E-G showed that the contents of anthocyanin and PA were significantly decreased in all transgenic plants compared to Col-0. However, the rutin content of lines At \#1 and At \#2 was significantly higher than Col-0, which was $109.8 \%$ and $119.3 \%$ of WT, respectively (Figure 4E-G). Collectively, these results suggest that ectopic expression of FtMYB3 inhibits anthocyanin/PA accumulation in plants. 

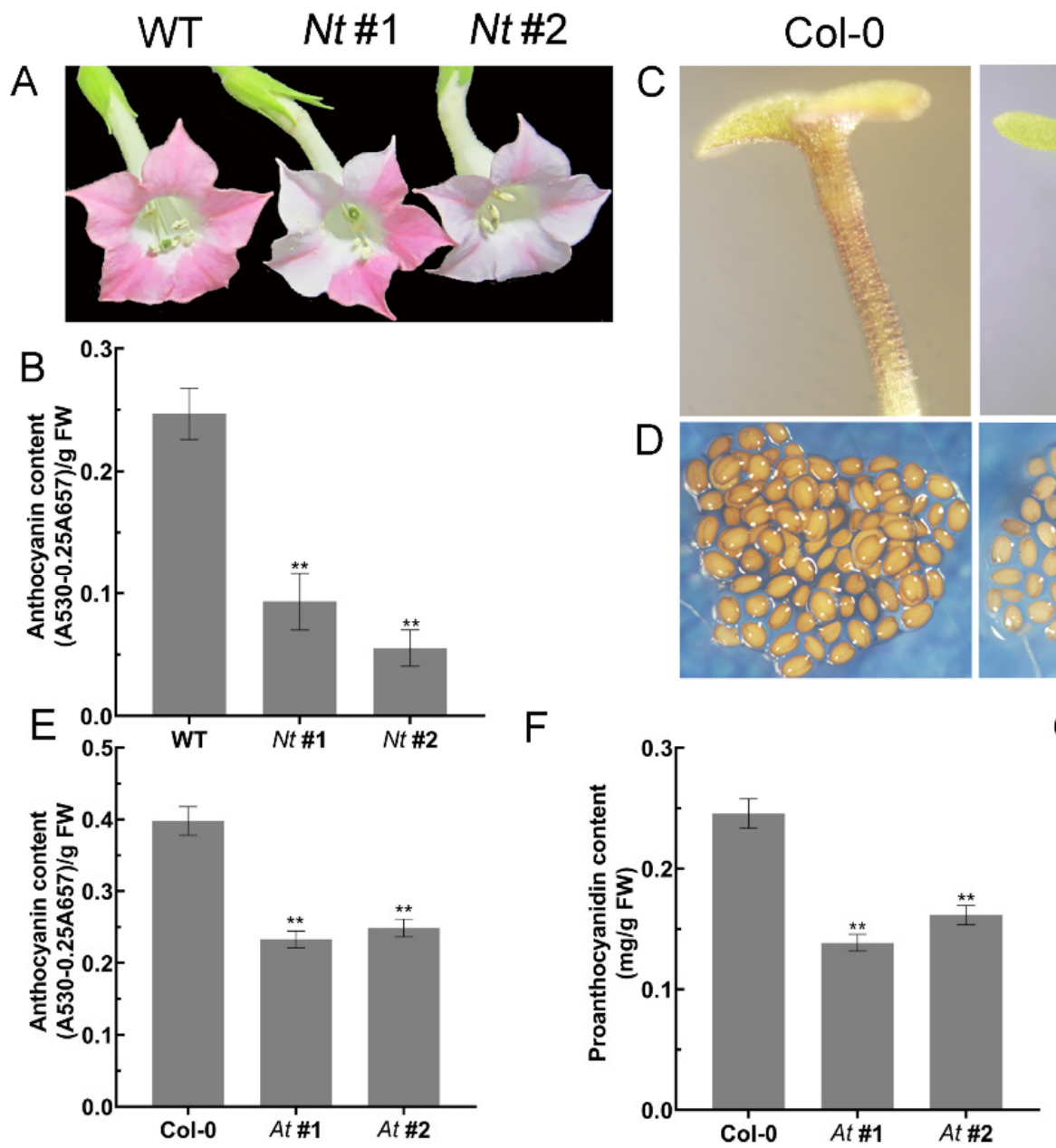

At\#1

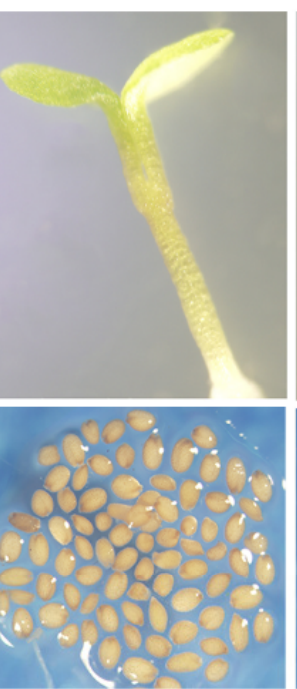

G

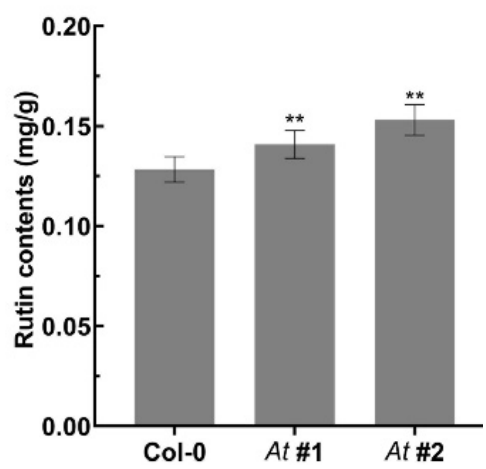

Figure 4. Phenotype of transgenic tobacco (termed $N t \# 1, N t \# 2$ ) and Arabidopsis (named At \#1, At \#2) overexpressing FtMYB3. (A,B) Floral phenotypes and total anthocyanin of WT and two transgenic $N t \# 1$ and $N t \# 2$ at the flowering stage; (C) Pigmentation of 6-day-old seedlings in Arabidopsis WT (termed Col-0) and transgenic lines $A t \# 1$ and $A t \# 2$ after $24 \mathrm{~h}$ treatment under $4{ }^{\circ} \mathrm{C}$; (D) Colour of seed coats in the same Arabidopsis as above without treatments; (E-G) Anthocyanin, PA and rutin content in the same Arabidopsis at the flowering phase. Error bars represent \pm SDs. ${ }^{* *}$ means extremely significant difference at $p<0.01$ level.

\subsection{Downregulation of FtMYB3 in the Expression Levels of DFR/BAN/ANS/TT13}

To elucidate the possible mechanisms of FtMYB3-mediated anthocyanin/PA biosynthesis, a qRT-PCR assay was performed to examine the effects of FtMYB3 on the transcript levels of genes functioning in the flavonoid biosynthetic pathway. Because of a long storage time, seeds of the previous transgenic tobacco failed to germinate. Thus, transgenic $A$. arabidopsis, At \#1 and At \#2 were used for subsequent experiments. qPCR results indicated that the expression levels of EBG genes ( $A t C H S, A t C H I$, and $A t F 3^{\prime} H$ ) were not significantly different in lines $A t \# 1$ and At \#2 compared to Col-0 (Figure 5). However, the transcript levels of LBG including DFR, ANS and BAN were significantly down-regulated in lines At\#1 and At \#2. Moreover, the transcript level of AtTT13, which is involved in PA transport [39], was also down-regulated. Interestingly, AtFLS showed an elevated expression level in line At \#2. These results indicated that FtMYB3 regulates multiple structural genes involved in flavonol biosynthesis. 

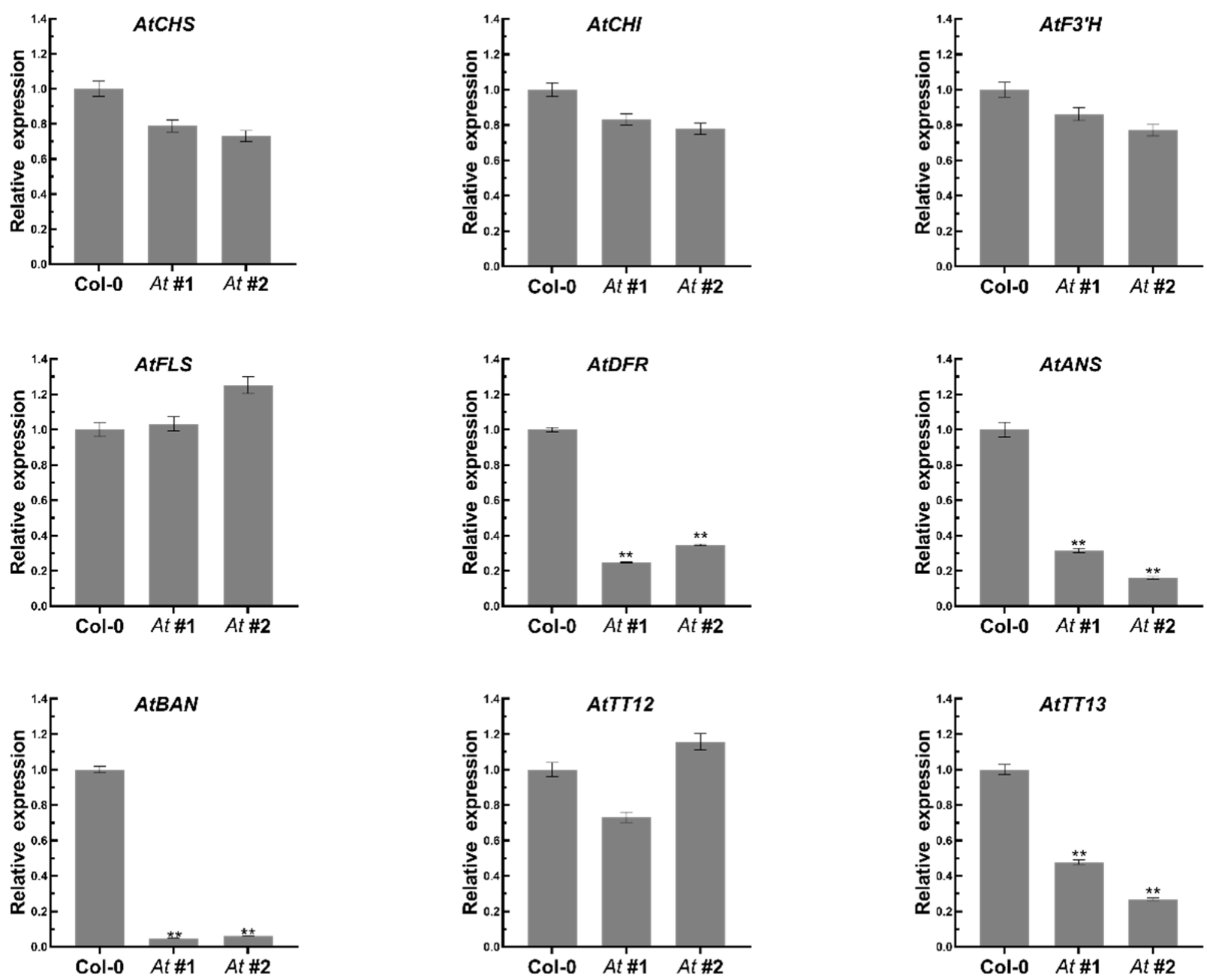

Figure 5. The expression level of enzyme genes in the flavonoid metabolic pathway in Arabidopsis overexpressing FtMYB3 (At \#1 and At \#2) compared with those in Col-0. The mRNA abundances of 9 genes were monitored by qRT-PCR in the 4-week-old seedings of Col-0, At \#1, and At \#2, respectively. The $2^{-\Delta \Delta C T}$ method was used to evaluate the relative expression, and AtActin2 was used as a reference gene. Error bars represent \pm SDs. ${ }^{* *}$ means extremely significant difference at $p<0.01$ level.

\subsection{FtMYB3 Interacts with GL3/EGL3/TT8/TTG1}

Previous studies have shown that MYB-bHLH-WD repeat (MBW) complexes regulate the transcription of anthocyanin genes in plants [27]. $\mathrm{Y} 2 \mathrm{H}$ was performed to screen interacting proteins of FtMYB3 involved in the formation of MBW complex. As shown in Figure 6, FtMYB3 can interact with GL3/EGL3/TT8/TTG1 in Tartary buckwheat and Arabidopsis, which suggests that FtMYB3 might regulate the transcriptional expression of key enzyme genes in the pathway of anthocyanin synthesis.

\subsection{FtMYB3 Inhibits the Transcriptional Activation Activity of the DFR Promoter}

$D F R$ is one of the essential enzyme genes for the synthesis of anthocyanin and flavonol branches. A transient expression assay was designed to test the impact of FtMYB3 on the transcriptional activation activity of $P_{A t D F R}$ and $P_{F t D F R 2}$. As shown in Figure 7 , GUS staining analysis showed that expression of the reporter gene declined significantly in the samples co-transformed with pCHF3-FtMYB3-eYFP. qRT-PCR detected the transcription level of the target gene GUS and showed that it had little or no transcriptional expression in the experimental group, suggesting that $F t M Y B 3$ indeed represses the transcriptional activation activity of $P_{A t D F R}$ and $P_{F t D F R 2}$. 

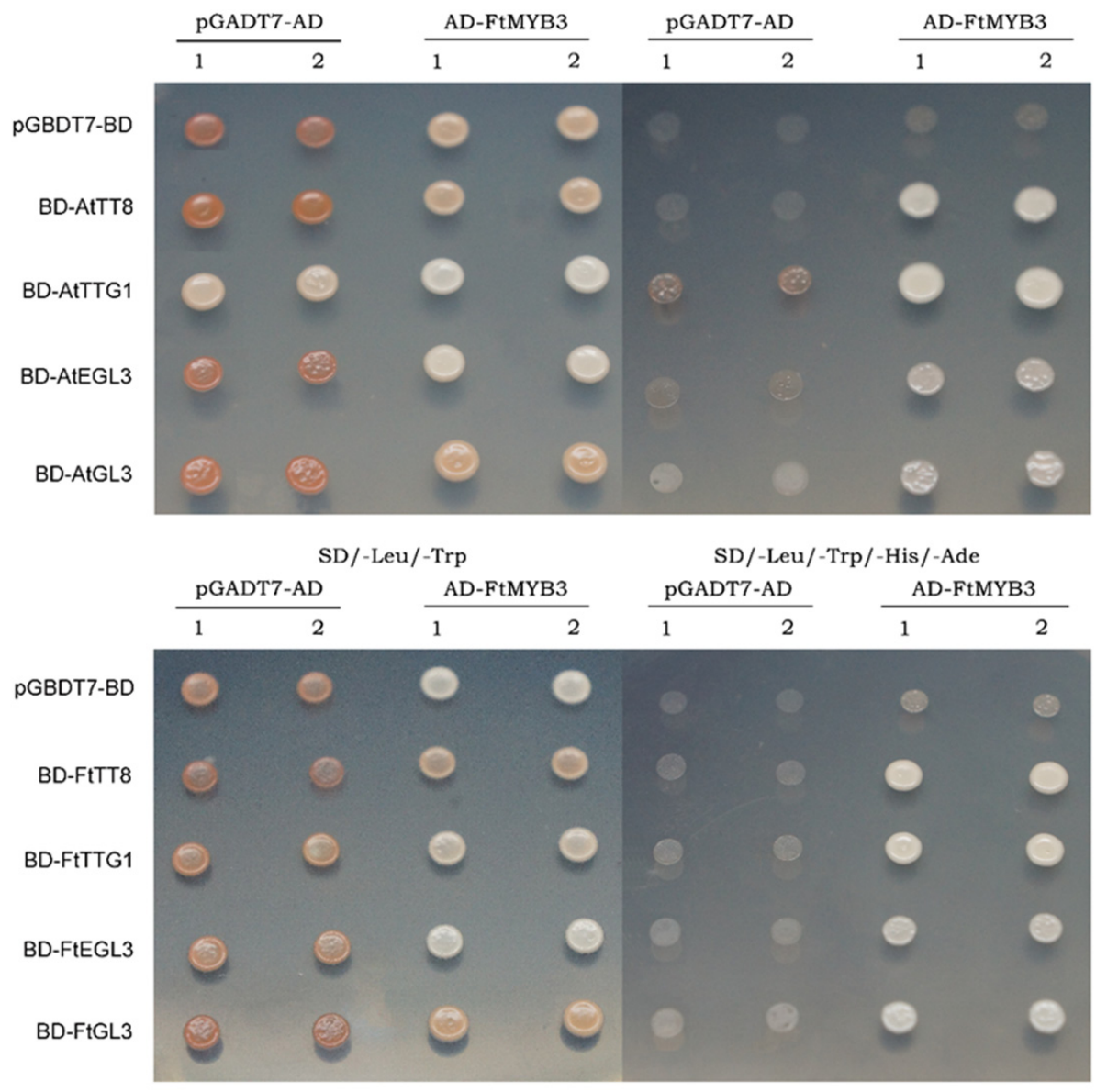

$\mathrm{SD} /$-Leu/-Trp

$\mathrm{SD} /$-Leu/-Trp/-His/-Ade

Figure 6. Interaction proteins of FtMYB3. FtMYB3 was fused to the pGADT7 vector, and FtTT8, FtTTG1, FtEGL3, or FtGL3 was fused to the pGBKT7 vector. SD/-Trp-Leu medium was used to screen the transformation for plasmids, and SD/-Leu-Leu-His-Ade medium was used to screen the transcriptional activation of HIS3 gene. Colony growth was monitored after 7 days. Yeast cells transformed with the empty plasmids pGADT7 and pGBKT7 were used as controls, respectively. Two independent technical replicates of each strain were analyzed.
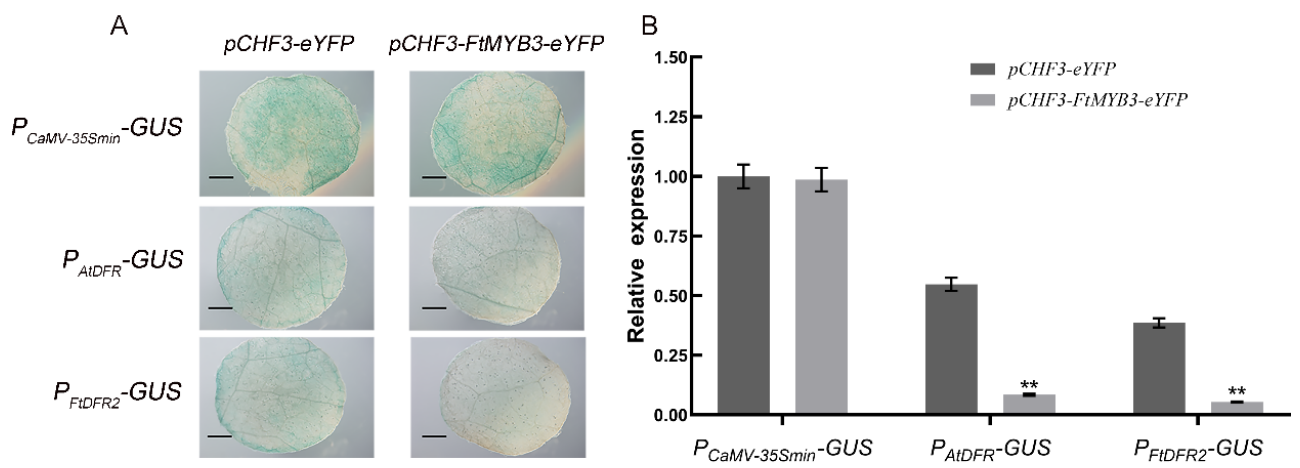

Figure 7. Inhibition of FtMYB3 on the DFR promoter in instantaneous transfected tobacco producing FtMYB3-eYFP together with the GUS gene under the control of the $P_{C a M V-35 S m i n}-G U S, P_{A t D F R}-G U S$ and $P_{\text {FtDFR2 }}$-GUS, respectively. (A) Staining of GUS protein. Tobacco leaves that were about 4 weeks old were used for transient transformation analysis, and GUS protein was stained $48 \mathrm{~h}$ after injection; (B) Relative expression of GUS gene. Error bars indicate \pm SDs. ${ }^{* *}$ means significant difference at $p<0.01$ level. 


\section{Discussion}

To date, most of the R2R3 MYB TFs have been well demonstrated to play a regulatory role in the flavonoid pathway in distinct plants. However, only two MYB repressors involved in the anthocyanin and PA biosynthesis were identified in Tartary buckwheat. In this study, we reported a novel R2R3-MYB TF FtMYB3 that negatively regulates anthocyanin and PA biosynthesis in a direct way.

\subsection{FtMYB3 Is a Special SG4 MYB Repressor That Reduces Anthocyanin and PA Accumulation}

In Arabidopsis, more than 100 R2R3-MYB TFs were divided into 25 sub-groups (SGs) [40]. Numerous R2R3-MYB TFs have been shown to be involved in the control of plant primary and secondary metabolism. Notably, SG4 and SG4-like are major TFs coordinating anthocyanin and PA biosynthesis. SG4 contains the conserved C2 motif that represses phenylpropanoid, sinapate ester, and flavonoid biosynthesis, while SG4-like contains the conserved $\mathrm{C} 5$ motif that participates in anthocyanin and PA biosynthesis. For instance, it has been shown that overexpression of AtMYB3, a SG 4 MYB protein represses phenylpropanoids biosynthesis [35]. The phylogenetic analysis indicated that FtMYB3 is very closely related to AtMYB3. In this study, we found that both anthocyanin and PA contents were significantly lower in transgenic Arabidopsis than Col-0, which suggests that the FtMYB3 gene is also involved in the negative regulation of anthocyanin and PA. In addition, FtMYB3 is also a homologue to the apple MdMYB3. Differently, ectopic expression of MdMYB3 in tobacco promotes anthocyanin and flavonol accumulation, even though these three genes contain the conserved $\mathrm{C} 2$ motifs at the $\mathrm{C}$-terminus [36]. However, the functional differences among FtMYB3, MdMYB3, and AtMYB3 remain unclear.

In Tartary buckwheat, two SG4-like R2R3 TFs FtMYB8 and FtMYB18 play a role in the synthesis of anthocyanins and Pas [31,32]. Their C-terminus contains a C5 motif, which is identified as the key to inhibiting the synthesis of Tartary buckwheat anthocyanins and Pas [31]. Similarly, other SG4-like also have an inhibitory effect on anthocyanin and PA bio-synthesis. For example, MrMYB6 might negatively regulate anthocyanin and PA accumulation in Chinese bayberry [41]. A similar finding was observed when PtrMYB57 was overexpressed in poplar [42]. Interestingly, we have observed a significant increase in rutin content in Arabidopsis upon overexpression of FtMYB3, indicating that other substrates can be involved in rutin synthesis. Many studies have reported a competitive relationship between the synthesis of anthocyanins and flavonols [43,44]. Overall, these results reveal that FtMYB3 may be a repressor for anthocyanin and PA biosynthesis.

\subsection{FtMYB3 Specifically Represses the Expression of Genes in the Late Pathway of Flavonoid Synthesis}

Previous studies have shown that the accumulation of anthocyanins and PAs are controlled by transcriptional regulation of genes encoding the early and late biosynthetic enzymes. In Ginkgo biloba, GbMYBF2 specifically inhibited the flux of flavonoid by repressing the transcription of $C H S, F 3 H, F L S$, and $A N S$, resulting in a decrease in anthocyanin contents [45]. Similarly, a C2 suppressor NtMYB2 from Chinese Narcissus inhibits the expression of key enzyme genes such as CHS, F3H, DFR, FLS, and ANS in transgenic tobacco, subsequently inhibiting the synthesis of anthocyanin and flavonol [46]. Moreover, FtMYB18 from Tartary buckwheat regulates anthocyanin synthesis by inhibition of EBGs (FtCHS) and LBGs (FtDFR) [31]. However, the inhibition of FtMYB8 in TTI12 to regulate anthocyanin/PA synthesis was mediated by anthocyanin/PA transport rather than anthocyanin/PA synthesis-related genes [32]. In this study, FtMYB3 significantly repressed the transcription level of $L B G s$ (DFR, ANS and BAN) upon its overexpression in Arabidopsis $(p<0.01)$. Remarkably, we also noted the reduced transcript abundance of an anthocyanin transporter gene TT13, which functions as a proton pump in the tonoplast of seed coat endothelium cells [39]. Given that FtMYB3 may have unique downstream target genes, we speculate that FtMYB3 may have a distinct regulation mechanism compared with previous anthocyanin and PA inhibitors. 


\subsection{Possible Mechanism of FtMYB3 Inhibiting the Biosynthesis of Anthocyanins and PAs}

R2R3-MYB repressors mainly include two types, FaMYB1-like and AtMYB4-like [47]. FaMYB1-like repressors incorporate into or bind MBW complexes to repress genes. AtMYB4like repressors directly bind on the promoter of target genes to actively repress transcription through its $\mathrm{C}$ terminal motifs such as $\mathrm{C} 2, \mathrm{C} 3$ or $\mathrm{C} 4$. For example, MdMYB16 directly inhibited the expression of MdUFGT and MdANS via its C2 motif in apple [16]. The Arabidopsis R3 type MYB transcription factor AtCPC also forms a complex to occupy the PAP1/ 2 binding sites of proteins such as TTG1, GL3, and EGL3, which inhibits the expression of late enzyme genes in the anthocyanin synthesis pathway and reduces anthocyanin synthesis and accumulation [48]. In this study, FtMYB3 was confirmed to interact with FtTT8, FtGL3, FtEGL3 and FtTTG1 in vitro, thereby inhibiting the transcriptional expression of vital enzyme genes in the anthocyanin pathway. We also found that FtMYB3 represses the transcriptional activation activity of $P_{A t D F R}$ and $P_{F t D F R 2}$, indicating that FtMYB3 may also directly target these genes. In addition, our previous studies have shown that FtMYB3 can interact with the jasmonate-ZIM domain protein FtJAZ2 to affect anthocyanin biosynthesis [33]. Notably, we found that FtMYB3 was strongly induced by MeJA. These results suggest that FtMYB3 regulates anthocyanin and PA biosynthesis through multiple pathways.

\section{Materials and Methods}

\subsection{Plant Materials and Growth Conditions}

The Tartary buckwheat "Xiqiao No. 2" used in this study is from Professor Anhu Wang of Xichang University. The A. thaliana ecotype Columbia- 0 (Col-0) was provided by Professor Yi Cai of Sichuan Agricultural University and the Nicotiana tabacum (NC89) was obtained from Professor Jinwen Zhang of Gansu Agricultural University. Different tissues (roots, stems, leaves, flowers, and seeds) from "Xiqiao No. 2" were collected at the flowering stage. Three biological replicates were prepared for each sample and immediately frozen in liquid nitrogen at $-80^{\circ} \mathrm{C}$ for storage.

\subsection{Total RNA Extraction and Gene Expression Analysis}

Total RNA from various plants was extracted using the EASYspin Plant RNA Kit (Aidlab, Beijing, China). Elimination of genomic DNA and the synthesis of first-strand cDNA were performed using the PrimeScript ${ }^{\mathrm{TM}}$ RT reagent Kit with gDNA Eraser (Takara, Dalian, China). qRT-PCR was conducted using SYBR ${ }^{\circledR}$ Premix Ex TaqTM II (Takara Bio Inc., Dalian, China), and the PCR program was as follows: one cycle of $3 \mathrm{~min}$ at $95^{\circ} \mathrm{C}$, followed by 40 cycles of $10 \mathrm{~s}$ at $95^{\circ} \mathrm{C}$ and $30 \mathrm{~s}$ at $60^{\circ} \mathrm{C}$. Three genes, FtH3, AtActin 2 and Nt $\beta$-actin were used as an internal control for Tartary buckwheat, Arabidopsis and tobacco, respectively. The relative expression level of these genes in this study was calculated using the Change $2^{(-\Delta \Delta C t)}$ method. All analyses were conducted with three biological replicates for each sample. Primer sequences used for qRT-PCR are listed in Table S2.

\subsection{Sequence Analysis of FtMYB3 and Its Promoter}

The full-length open reading frame (ORF) of FtMYB3 was obtained by PCR using gene-specific primers in terms of the published sequence (GenBank ID: JF313349). The molecular weight, isoelectric point (pI), and theoretical coding sequence of FtMYB3 were predicted using an online ExPASy server: https:/ / web.expasy.org/protparam/ (accessed on 7 January 2022). The protein sequences of R2R3-MYB transcriptional factors from other species were collected from NCBI: https:/ / blast.ncbi.nlm.nih.gov / Blast.cgi (accessed on 20 August 2020). Subsequently, these sequences were aligned with FtMYB3 by the DNAMAN6.0 software with default parameters. The entire protein sequence of R2R3-MYB TFs related to FtMYB3 were used to create a phylogenetic tree using MEGA5 software with default parameters. The bootstrap was 1000 . 


\subsection{Construction of Expression Vectors and Stable Transformation of Arabidopsis and Tobacco}

To obtain the vector $\mathrm{PCHF3-FtMYB3-eYFP,} \mathrm{PCR} \mathrm{was} \mathrm{performed} \mathrm{with} \mathrm{primers} \mathrm{FtMYB3F}$ and FtMYB3R using cDNA as a template. The cDNA was synthesized based on RNA extracted from young leaves of "Xiqiao No. 2". PCR amplification was performed using the KOD-plus-neo high fidelity enzyme kit (Toyobo, Osaka, Japan). The PCR fragment without the stop codon encoding the whole coding sequences of FtMYB3 was inserted between Kpn I and Sal I sites in plasmid pCHF3-YFP that was provided by Professor Yi Cai of Sichuan Agricultural University. Subsequently, the pCHF3-FtMYB3-eYFP vector was introduced into Col-0 through Agrobacterium tumefaciens GV3101 by the floral dip method [49]. Meanwhile, pCHF3-FtMYB3-YFP was transformed into NC89 by using the Agrobacterium strain LBA4404 according to the method described by Huang [32]. The first generation (T1) seeds of transgenic plants were collected and screened in 1/2 MS medium with $50 \mathrm{mg}$ L-1 kanamycin. Next, the kanamycin-resistant plants were transferred into soil pots and grown in a growth chamber with a $16 \mathrm{~h}$ photoperiod cycle at $25^{\circ} \mathrm{C}$. Similarly, the second generation (T2) seeds were collected. Finally, the T3 homozygous positive lines were collected and used for subsequent experiments. Primer sequences used for constructing the expression vector are listed in Table S2.

\subsection{Determination of Total Anthocyanin, PA and Rutin Content}

Total anthocyanins were extracted and quantified in fresh leaves of transgenic Arabidopsis using the previously described method in [50] as follows: (1) the samples were ground in liquid nitrogen to produce powder; (2) $200 \mathrm{mg}$ powder were used to extract the anthocyanins in $1 \mathrm{~mL}$ of an acidic methanol solution; (3) the above solution was sedimented by centrifugation $\left(12,000 \times g, 4^{\circ} \mathrm{C}, 1 \mathrm{~min}\right)$ and $400 \mu \mathrm{L}$ of the supernatant was added to $600 \mu \mathrm{L}$ of acidic methanol and then filtered over a $0.2 \mu \mathrm{m}$ Teflon filter before analysis; and (4) the anthocyanins were then measured at 530 and $657 \mathrm{~nm}$ in a spectrometer (UNICO WFJ2000, China). The extraction and measurement of Pas were carried out as previously described in [51]. High-performance liquid chromatography (HPLC) was performed to determine the rutin content according to the description from Yao [52].

\subsection{Yeast Two-Hybrid Assay (Y2H)}

For $\mathrm{Y} 2 \mathrm{H}$ assay, the full-length CDS of FtMYB3 was inserted into the pGADT7 vector as a bait vector. Meanwhile, the full-length CDS of AtGL3, AtEGL3, AtTTG1, FtTT8, FtGL3, FtEGL3 and FtTTG1 was inserted into the pGBKT7 vector as a prey vector. Subsequently, the prey and the bait constructs were transformed into yeast strain AH109 (Clontech) [53]. The yeast cells were grown on SD/-Trp-Leu medium after mating. Positive colonies were confirmed by PCR and further grown on SD/-Trp-Leu-His-Ade defective medium. Pictures were captured on day 3 after incubation. pGADT7 and pGBKT7 were provided by Professor Yi Cai of Sichuan Agricultural University. All primers used for PCR are listed in Table S2.

\subsection{Transient Expression Assay}

The promoter of FtDFR2 and AtDFR was inserted into the plant expression vector pCAMBIA1304 by replacing the 35S promoter before the GUS gene, respectively. The recombinant vector pCAMBIA1304- $P_{\text {FtDFR2 }}-G U S$ (termed $P_{F t D F R 2}-G U S$ ) and pCAMBIA1304$P_{A t D F R}$-GUS (termed $P_{A t D F R}-G U S$ ) were transformed into the GV3101 and then injected into the young leaves of tobacco expressing pCHF3-eYFP or pCHF3-FtMYB3-eYFP vectors, respectively. Lastly, the expression of GUS gene was detected by qRT-PCR and visualized by histochemical staining after $48 \mathrm{~h}$ incubation.

\subsection{Statistical Analysis}

Statistical analyses were conducted by SPSS 22.0 software. All data were obtained from three biological replicates. ${ }^{*}$ and ${ }^{* *}$ indicate $p$-values $<0.05$ and $<0.01$, respectively. 


\section{Conclusions}

In this study, our data indicate that FtMYB3 is an R2R3 inhibitor that negatively regulates the anthocyanin and PA accumulation by the strong downregulation of DFR, ANS, BAN and TT13. Therefore, FtMYB3 may be a potential target gene for improving the quality of Tartary buckwheat. Furthermore, we found that FtMYB3 can directly interact with TT8, GL3, EGL3, and TTG1 in vitro. Based on these results, we propose a putative model in which FtMYB3 accumulation leads to decreased expression of DFR and ANS and thus reduces anthocyanin and PA content (Figure 8). Taken together, this study promotes further understanding of the molecular mechanisms of regulation for anthocyanin and PA synthesis in Tartary buckwheat.

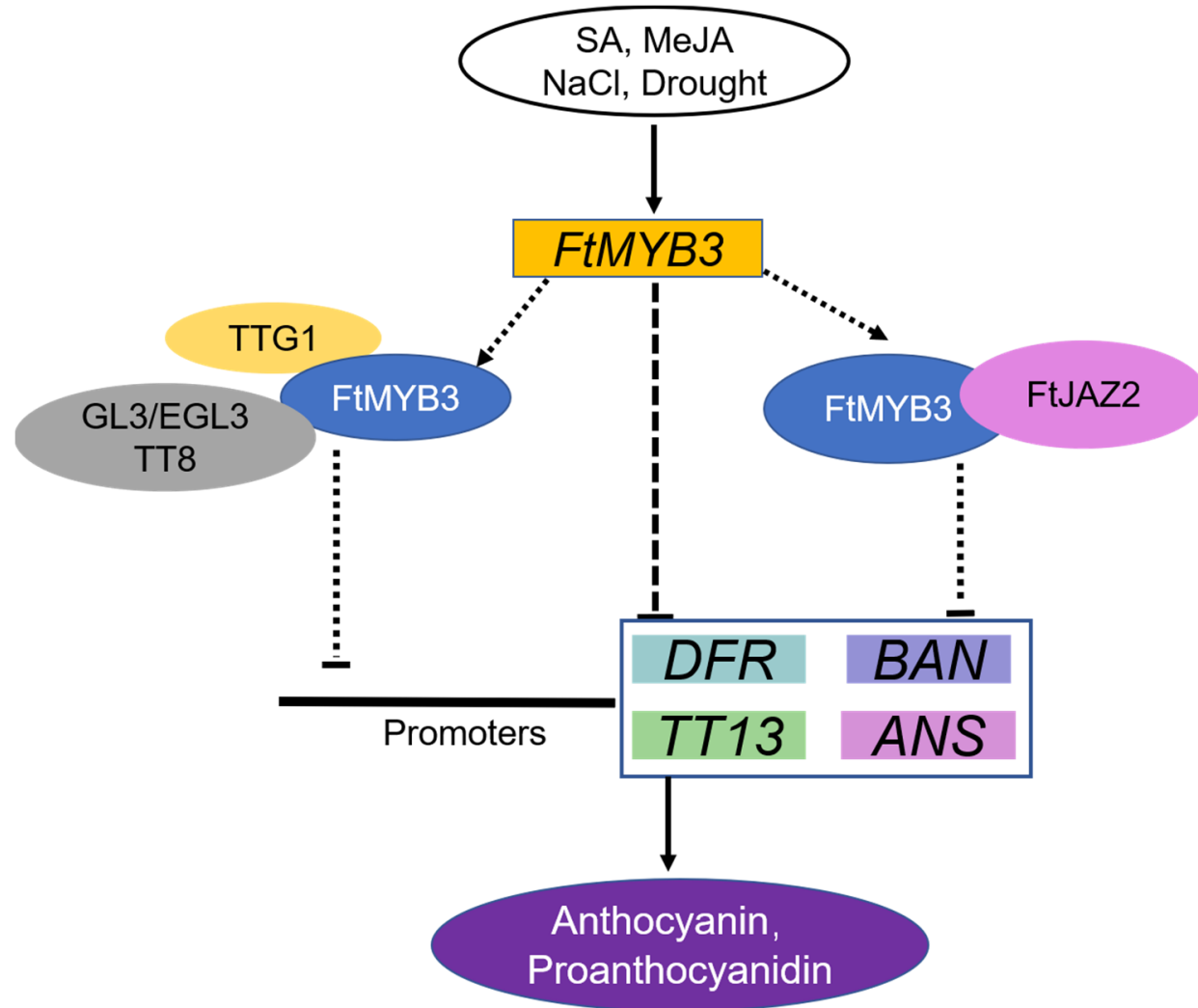

Figure 8. A proposed model depicting FtMYB3 regulation of anthocyanin and PA synthesis under different stresses. FtMYB3 is induced by SA, MeJA, $\mathrm{NaCl}$ and drought stress. FtMYB3 directly interacts with TT8, GL3, EGL3, and TTG1 and suppresses their transcriptional activator of DFR, ANS, $B A N$ and TT13. Additionally, FtMYB3 may inhibit the synthesis of anthocyanins/PAs by interacting with FtJAZ2 protein.

Supplementary Materials: The following supporting information can be downloaded at: https: //www.mdpi.com/article/10.3390/ijms23052775/s1.

Author Contributions: Conceptualization, H.Z. and Q.W.; methodology, R.D. and Y.B.; software, L.W. and C.L.; formal analysis, H.W.; writing-original draft preparation, L.W. All authors have read and agreed to the published version of the manuscript.

Funding: This research was funded by the National Natural Science Foundation of China, grant number 31871698.

Institutional Review Board Statement: Not applicable.

Informed Consent Statement: Not applicable.

Data Availability Statement: Not applicable. 
Acknowledgments: The authors would also like to thank Anhu Wang for the "Xiqiao No. 2" seeds.

Conflicts of Interest: The authors declare no conflict of interest.

\section{References}

1. Tanaka, Y.; Sasaki, N.; Ohmiya, A. Biosynthesis of plant pigments: Anthocyanins, betalains and carotenoids. Plant J. 2010, 54, 733-749. [CrossRef] [PubMed]

2. Liu, Y.; Tikunov, Y.; Schouten, R.E.; Marcelis, L.; Visser, R.; Arnaud, B. Anthocyanin Biosynthesis and Degradation Mechanisms in Solanaceous Vegetables: A Review. Front. Chem. 2018, 6, 52. [CrossRef]

3. Lloyd, A.; Brockman, A.; Aguirre, L.; Annabelle, C.; Alex, B.; Araceli, C.; Antonio, G. Advances in the MYB-bHLH-WD Repeat (MBW) Pigment Regulatory Model: Addition of a WRKY Factor and Co-option of an Anthocyanin MYB for Betalain Regulation. Plant Cell Physiol. 2017, 58, 1431-1441. [CrossRef]

4. $\quad$ Li, D.; Wang, P.; Luo, Y.; Zhao, M.; Chen, F. Health benefits of anthocyanins and molecular mechanisms: Update from recent decade. Crit. Rev. Food Sci. 2017, 57, 1729-1741. [CrossRef] [PubMed]

5. Zhu, F. Proanthocyanidins in cereals and pseudocereals. Crit. Rev. Food Sci. Nutr. 2019, 59, 1521-1533. [CrossRef]

6. Springob, K.; Nakajima, J.; Yamazaki, M.; Saito, K. Recent advances in the biosynthesis and accumulation of anthocyanins. Nat. Prod. Rep. 2003, 20, 288-303. [CrossRef]

7. Li, P.; Dong, Q.; Ge, S.; He, X.; Verdier, J.; Li, D.; Zhao, J. Metabolic engineering of proanthocyanidin production by repressing the isoflavone pathways and redirecting anthocyanidin precursor flux in legume. Plant Biotechnol. J. 2016, 14, 1604-1618. [CrossRef]

8. Xie, D.; Sharma, S.; Paiva, N.; Ferreira, D.; Dixon, R. Role of anthocyanidin reductase, encoded by BANYULS in plant flavonoid biosynthesis. Science 2003, 299, 396-399. [CrossRef] [PubMed]

9. Jaakola, L. New insights into the regulation of anthocyanin biosynthesis in fruits. Trends Plant Sci. 2013, 18, 477-483. [CrossRef]

10. Li, C.; Wu, J.; Hu, K.D.; Wei, H.; Sun, H.; Hu, L.; Han, Z.; Yao, G.; Zhang, H. PyWRKY26 and PybHLH3 cotargeted the PyMYB114 promoter to regulate anthocyanin biosynthesis and transport in red-skinned pears. Hortic. Res. 2020, 7, 37. [CrossRef]

11. Marles, M.; Ray, H.; Gruber, M. New perspectives on proanthocyanidin biochemistry and molecular regulation. Phytochemistry 2003, 64, 367-383. [CrossRef]

12. Zhang, Z.; Tian, C.; Zhang, Y.; Li, C.; Li, X.; Yu, Q.; Wang, S.; Wang, X.; Chen, X.; Feng, X. Transcriptomic and metabolomic analysis provides insights into anthocyanin and procyanidin accumulation in pear. BMC Plant Biol. 2020, 20, 129. [CrossRef] [PubMed]

13. Tian, J.; Zhang, J.; Han, Z.; Song, T.; Li, J.; Wang, Y.; Yao, Y. McMYB12 Transcription Factors Co-regulate Proanthocyanidin and Anthocyanin Biosynthesis in Malus Crabapple. Sci. Rep. 2017, 7, 43715. [CrossRef]

14. An, X.; Tian, Y.; Chen, K.; Liu, X.; Liu, D.; Xie, X.; Cheng, C.; Cong, P.; Hao, Y. MdMYB9 and MdMYB11 are involved in the regulation of the JA-induced biosynthesis of anthocyanin and proanthocyanidin in apples. Plant Cell Physiol. 2015, 56, 650-662. [CrossRef] [PubMed]

15. Anwar, M.; Yu, W.; Yao, H.; Zhou, P.; Allan, A.; Zeng, L. NtMYB3, an R2R3-MYB from Narcissus, Regulates Flavonoid Biosynthesis. Int. J. Mol. Sci. 2019, 20, 5456. [CrossRef]

16. Xu, H.; Wang, N.; Liu, J.; Qu, C.; Wang, Y.; Jiang, S.; Lu, N.; Wang, D.; Zhang, Z.; Chen, X. The molecular mechanism underlying anthocyanin metabolism in apple using the MdMYB16 and MdbHLH33 genes. Plant Mol. Biol. 2017, 94, 149-165. [CrossRef]

17. Cheng, J.; Yu, K.; Shi, Y.; Wang, J.; Duan, C. Transcription Factor VviMYB86 Oppositely Regulates Proanthocyanidin and Anthocyanin Biosynthesis in Grape Berries. Front Plant Sci. 2020, 11, 2263. [CrossRef]

18. Liu, Y.; Hou, H.; Jiang, X.; Wang, P.; Dai, X.; Chen, W.; Gao, L.; Xia, T. A WD40 Repeat Protein from Camellia sinensis Regulates Anthocyanin and Proanthocyanidin Accumulation through the Formation of MYB-bHLH-WD40 Ternary Complexes. Int. J. Mol. Sci. 2018, 19, 1686. [CrossRef]

19. Gonzalez, A.; Zhao, M.; Leavitt, J.; Lloyd, A. Regulation of the anthocyanin biosynthetic pathway by the TTG1/bHLH/Myb transcriptional complex in Arabidopsis seedlings. Plant J. 2008, 53, 814-827. [CrossRef]

20. An, J.; Xu, R.; Liu, X.; Zhang, J.; Wang, X.; You, C.; Hao, Y. Jasmonate induces anthocyanin and proanthocyanidin biosynthesis in apple by mediating the JAZ1-TRB1-MYB9 complex. Plant J. 2021, 106, 1414-1430. [CrossRef]

21. Mao, Z.; Jiang, H.; Wang, S.; Wang, Y.; Yu, L.; Zou, Q.; Liu, W.; Jiang, S.; Wang, N.; Zhang, Z.; et al. The MdHY5-MdWRKY41MdMYB transcription factor cascade regulates the anthocyanin and proanthocyanidin biosynthesis in red-fleshed apple. Plant Sci. 2021, 306, 110848. [CrossRef]

22. Li, G.; Meng, X.; Zhu, M.; Li, Z. Research Progress of Betalain in Response to Adverse Stresses and Evolutionary Relationship Compared with Anthocyanin. Molecules 2019, 24, 3078. [CrossRef]

23. Yu, D.; Huang, T.; Tian, B.; Zhan, J. Advances in Biosynthesis and Biological Functions of Proanthocyanidins in Horticultural Plants. Foods 2020, 9, 1774. [CrossRef]

24. Joshi, D.C.; Zhang, K.; Wang, C.; Chandora, R.; Khurshid, M.; Li, J.; He, M.; Georgiev, M.; Zhou, M. Strategic enhancement of genetic gain for nutraceutical development in buckwheat: A genomics-driven perspective. Biotechnol. Adv. 2020, $39,107479$. [CrossRef] [PubMed]

25. Zhu, F. Chemical composition and health effects of Tartary buckwheat. Food Chem. 2016, 203, 231-245. [CrossRef] [PubMed] 
26. Cirillo, V.; D’Amelia, V.; Esposito, M.; Amitrano, C.; Carillo, P.; Carputo, D.; Maggio, A. Anthocyanins are Key Regulators of Drought Stress Tolerance in Tobacco. Biology 2021, 10, 139. [CrossRef] [PubMed]

27. Naing, A.; Kim, C. Abiotic stress-induced anthocyanins in plants: Their role in tolerance to abiotic stresses. Physiol. Plant. 2021, 172, 1711-1723. [CrossRef]

28. Malisch, C.; Salminen, J.; Kölliker, R.; Engström, M.; Suter, D.; Studer, B.; Lüscher, A. Drought Effects on Proanthocyanidins in Sainfoin (Onobrychis viciifolia Scop.) Are Dependent on the Plant's Ontogenetic Stage. J. Agric. Food Chem. 2016, 64, 9307-9316. [CrossRef]

29. Bai, Y.; Li, C.; Zhang, J.; Li, S.; Luo, X.; Yao, H.; Chen, H.; Zhao, H.; Park, S.U.; Wu, Q. Characterization of two tartary buckwheat R2R3-MYB transcription factors and their regulation of proanthocyanidin biosynthesis. Physiol. Plant. 2014, 152, 431-440. [CrossRef]

30. Luo, X.; Zhao, H.; Yao, P.; Li, Q.; Huang, Y.; Li, C.; Chen, H.; Wu, Q. An R2R3-MYB Transcription Factor FtMYB15 Involved in the Synthesis of Anthocyanin and Proanthocyanidins from Tartary Buckwheat. J. Plant Growth Regul. 2017, 37, 76-84. [CrossRef]

31. Dong, Q.; Zhao, H.; Huang, Y.; Chen, Y.; Wan, M.; Zeng, Z.; Yao, P.; Li, C.; Wang, X.; Chen, H.; et al. FtMYB18 acts as a negative regulator of anthocyanin/proanthocyanidin biosynthesis in Tartary buckwheat. Plant Mol. Biol. 2020, 104, 309-325. [CrossRef] [PubMed]

32. Huang, Y.; Wu, Q.; Wang, S.; Shi, J.; Dong, Q.; Yao, P.; Shi, G.; Xu, S.; Deng, R.; Li, C.; et al. FtMYB8 from Tartary buckwheat inhibits both anthocyanin/Proanthocyanidin accumulation and marginal Trichome initiation. BMC Plant Biol. 2019, 19, 263. [CrossRef]

33. Luo, X.; Li, S.; Yao, P.; Li, C.; Chen, H.; Wu, Q.; Zhao, H. The jasmonate-ZIM domain protein FtJAZ2 interacts with the R2R3MYBtranscription factor FtMYB3 to affect anthocyanin biosynthesis in tartary buckwheat. Turk. J. Biol. 2017, 41, 526-534. [CrossRef]

34. Li, X.; Sathasivam, R.; Park, N.I.; Wu, Q.; Park, S. Enhancement of phenylpropanoid accumulation in tartary buckwheat hairy roots by overexpression of MYB transcription factors. Ind. Crops Prod. 2020, 156, 112887. [CrossRef]

35. Zhou, M.; Zhang, K.; Sun, Z.; Yan, M.; Chen, C.; Zhang, X.; Tang, Y.; Wu, Y. LNK1 and LNK2 Corepressors Interact with the MYB3 Transcription Factor in Phenylpropanoid Biosynthesis. Plant Physiol. 2017, 174, 1348-1358. [CrossRef]

36. Vimolmangkang, S.; Han, Y.; Wei, G.; Korban, S. An apple MYB transcription factor, MdMYB3, is involved in regulation of anthocyanin biosynthesis and flower development. BMC Plant Biol. 2013, 13, 176. [CrossRef]

37. Grotewold, E.; Sainz, M.B.; Tagliani, L.; Hernandez, J.M.; Chandler, V.L. Identification of the residues in the Myb domain of maize $\mathrm{C} 1$ that specify the interaction with the bHLH cofactor R. Proc. Natl. Acad. Sci. USA 2000, 97, 13579-13584. [CrossRef]

38. Fang, H.; Dong, Y.; Yue, X.; Hu, J.; Jiang, S.; Xu, H.; Wang, Y.; Su, M.; Zhang, J.; Zhang, Z. The B-box zinc finger protein MdBBX20 integrates anthocyanin accumulation in response to ultraviolet radiation and low temperature. Plant Cell Environ. 2019, 42, 2090-2104. [CrossRef]

39. Appelhagen, I.; Nordholt, N.; Seidel, T.; Spelt, K.; Koes, R.; Quattrochio, F.; Sagasser, M.; Weisshaar, B. TRANSPARENT TESTA 13 is a tonoplast P3A -ATPase required for vacuolar deposition of proanthocyanidins in Arabidopsis thaliana seeds. Plant J. 2015, 82, 840-849. [CrossRef] [PubMed]

40. Dubos, C.; Stracke, R.; Grotewold, E.; Weisshaar, B.; Martin, C.; Lepiniec, L. MYB transcription factors in Arabidopsis. Trends Plant Sci. 2010, 15, 573-581. [CrossRef] [PubMed]

41. Shi, L.; Chen, X.; Wang, K.; Yang, M.; Chen, W.; Yang, Z.; Gao, S. MrMYB6 from Chinese Bayberry (Myrica rubra) Negatively Regulates Anthocyanin and Proanthocyanidin Accumulation. Front. Plant Sci. 2021, 12, 1218. [CrossRef]

42. Wan, S.; Li, C.; Ma, X.; Luo, K. PtrMYB57 contributes to the negative regulation of anthocyanin and proanthocyanidin biosynthesis in poplar. Plant Cell Rep. 2017, 36, 1263-1276. [CrossRef]

43. Davies, K.M.; Schwinn, K.E.; Deroles, S.C.; Manson, D.G.; Bradley, J.M. Enhancing anthocyanin production by altering competition for substrate between flavonol synthase and dihydroflavonol 4-reductase. Euphytica 2003, 131, 259-268. [CrossRef]

44. Yuan, Y.; Rebocho, A.B.; Sagawa, J.M.; Stanley, L.E.; Bradshaw, H.D. Competition between anthocyanin and flavonol biosynthesis produces spatial pattern variation of floral pigments between Mimulus species. Proc. Natl. Acad. Sci. USA 2016, 113, 2448-2453. [CrossRef]

45. Xu, F.; Ning, Y.; Zhang, W.; Liao, Y.; Li, L.; Cheng, H.; Cheng, S. An R2R3-MYB transcription factor as a negative regulator of the flavonoid biosynthesis pathway in Ginkgo biloba. Funct. Integr. Genom. 2014, 14, 177-189. [CrossRef] [PubMed]

46. Muhammad, A.; Wang, G.; Wu, J.; Saquib, W.; Andrew, A.; Zeng, L. Ectopic Overexpression of a Novel R2R3-MYB, NtMYB2 from Chinese Narcissus Represses Anthocyanin Biosynthesis in Tobacco. Molecules 2018, 23, 781

47. Chen, L.; Hu, B.; Qin, Y.; Hu, G.; Zhao, J. Advance of the negative regulation of anthocyanin biosynthesis by MYB transcription factors. Plant Physiol. Bioch. 2019, 136, 178-187. [CrossRef] [PubMed]

48. Zhu, H.; Fitzsimmons, K.; Khandelwal, A.; Kranz, R.G. CPC, a single-repeat R3 MYB, is a negative regulator of anthocyanin biosynthesis in Arabidopsis. Mol. Plant 2009, 2, 790-802. [CrossRef]

49. Clough, S.J.; Bent, A.F. Floral Dip: A Simplified Method for Agrobacterium-mediated Transformation of Arabidopsis Thaliana Plant J. 1998, 16, 735-743. [CrossRef]

50. Zhang, W.; Ning, G.; Haiyan, L.; Liao, L.; Bao, M. Single MYB-type transcription factor AtCAPRICE: A new efficient tool to engineer the production of anthocyanin in tobacco. Biochem. Biophys. Res. Commun. 2009, 388, 742-747. [CrossRef] [PubMed] 
51. Peel, G.J.; Dixon, R. A Detection and Quantification of Engineered Proanthocyanidins in Transgenic Plants. Nat. Prod. Commun. 2007, 2, 1009-1014. [CrossRef]

52. Yao, P.; Huang, Y.; Dong, Q.; Wan, M.; Wang, A.; Chen, Y.; Li, C.; Wu, Q.; Chen, H.; Zhao, H. FtMYB6, a Light-Induced SG7 R2R3-MYB Transcription Factor, Promotes Flavonol Biosynthesis in Tartary Buckwheat (Fagopyrum tataricum). J. Agric. Food Chem. 2020, 68, 13685-13696. [CrossRef] [PubMed]

53. James, P.; Halladay, J.; Craig, E.A. Genomic Libraries and a Host Strain Designed for Highly Efficient Two-Hybrid Selection in Yeast. Genetics 1996, 144, 1425-1436. [CrossRef] [PubMed] 